\title{
ON A CLASS OF POLYNOMIALS OBTAINED FROM GENERALIZED HUMBERT POLYNOMIALS
}

\author{
DAVID ZEITLIN
}

1. Introduction. Recently, Gould [1] defined a generalized Humbert polynomial, $P_{n}(m, x, y, p, C)$, of degree $n$ in $x$, by the series expansion

$$
\left(C-m x t+y t^{m}\right)^{p}=\sum_{n=0}^{\infty} t^{n} P_{n}(m, x, y, p, C),
$$

where $m \geqq 1$ is an integer and the other parameters, $C, x, y$, and $p$, are unrestricted in general. By a proper choice of parameters, one obtains from (1.1) the generating function for such polynomials as Gegenbauer, Legendre, Humbert $\left(P_{n}(m, x, 1,-p, 1)\right)$, and Chebyshev polynomials, $U_{n}(x)$, of the second kind, as important special cases. An extensive list of references is given in [1].

The aim of this paper is to give the important properties of the polynomials $R_{n}(x, m, C / y)$ which we define by the limit

$$
\begin{aligned}
& \lim _{\nu \rightarrow 0} P_{n}(m, x, y, p, C) / p \\
& \quad=-(m / n)(C / y)^{-n / 2} \cdot R_{n}\left(m x /\left(2(C y)^{1 / 2}\right), m, C / y\right) \quad(n \neq 0) .
\end{aligned}
$$

Noting that for $k>0, P_{n}(2, x, 1,-k, 1)=C_{n}^{(\boldsymbol{k})}(x)$, the Gegenbauer polynomial, and recalling $[2$, p. $82,(4.7 .8)]$ that $\lim _{k \rightarrow 0} C_{n}^{(\mathbf{k})}(x) / k$ $=(2 / n) T_{n}(x), n \neq 0$, where $T_{n}(x)$ is the Chebyshev polynomial of the first kind, it follows from $(1.2)$ that $R_{n}(x, 2,1) \equiv T_{n}(x)$, which is indeed the case (see (1.4)).

We shall cite only those results of [1] which are needed to make this paper self-contained. In $[1$, p. $699,(2.1)]$ it is shown that

$$
\begin{aligned}
P_{n}(m, x, y, p, C) & \\
= & \sum_{k=0}^{[n / m]}\left(\begin{array}{l}
p \\
k
\end{array}\right)\left(\begin{array}{c}
p-k \\
n-m k
\end{array}\right) C^{p-n+(m-1) k} y^{k}(-m x)^{n-m k} .
\end{aligned}
$$

Applying (1.2) to (1.3) and noting that

$$
\left(\begin{array}{l}
p \\
k
\end{array}\right)=p(p-1) \cdots(p-k+1) / k !
$$

Received by the editors May 3, 1966. 
we obtain

$$
\lim _{p \rightarrow 0}\left(\begin{array}{l}
p \\
k
\end{array}\right)\left(\begin{array}{c}
p-k \\
n-m k
\end{array}\right) / p=(-1)^{n-m k+k-1} \frac{[n-1-(m-1) k] !}{k !(n-m k) !} ;
$$

and hence, after simplification, with $b=C / y$, that

$$
\begin{array}{r}
R_{n}(x, m, b) \\
=\frac{n}{m} \sum_{k=0}^{[n / m]}(-1)^{k} \frac{[n-1-(m-1) k] !}{k !(n-m k) !} b^{(m-2) k / 2}(2 x)^{n-m k} \\
(n=1,2, \cdots) .
\end{array}
$$

It is convenient to define $R_{0}(x, m, b) \equiv 1$. We note that $R_{n}(x, 2, b)$ $=T_{n}(x)$, and $R_{n}(x, 1, b)=\left(2 x-b^{-\frac{1}{2}}\right)^{n}$.

The inverse relation to (1.4) is given by

$$
(2 x)^{n}=m \sum_{k=0}^{[n / m]}\left(\begin{array}{l}
n \\
k
\end{array}\right) b^{(m-2) k / 2} R_{n-m k}(x, m, b),
$$

which was obtained by applying (1.2) to the inverse relation of (1.3) (see $[1$, p. $707,(6.2)]$ )

$$
\begin{aligned}
\left(\begin{array}{l}
p \\
n
\end{array}\right)(-m x)^{n}= & \sum_{k=0}^{[n / m]}(-1)^{k}\left(\begin{array}{c}
p-n+k \\
k
\end{array}\right) \frac{p+m k-n}{p+k-n} \\
& \cdot C^{n-k-p} y^{k} P_{n-m k}(m, x, y, p, C) .
\end{aligned}
$$

For $m=2$, (1.5) reduces to the known relation for $T_{n}(x)$ (see, e.g., $[3$, p. 223, Problem $4 \mathrm{~b}])$. In the sequel, we shall write $R_{n}(x, m, b) \equiv R_{n}$ and $P_{n}(m, x, y, p, C) \equiv P_{n}$.

2. Properties of the polynomials. $R_{n}$ satisfies a linear difference equation of order $m$ with constant coefficients

$$
R_{n+m}-2 x R_{n+m-1}+b^{(m-2) / 2} R_{n}=0 \quad(n=0,1, \cdots),
$$

and from (1.4), $R_{n}=(2 x)^{n} / m, n=1,2, \cdots, m-1$, and $R_{0}=1$. For $m=2,(2.1)$ is the difference equation satisfied by Chebyshev polynomials. We obtained (2.1) by applying (1.2) to

$$
\begin{aligned}
& C n P_{n}-m(n-1-p) x P_{n-1}+(n-m-m p) y P_{n-m}=0 \\
&(n \geqq m \geqq 1),
\end{aligned}
$$

(see $[1$, p. $700,(2.3)])$. Applying a well-known result (see $[4$, p. 300]) for generating functions, i.e., if $Y_{0}, Y_{1}, \cdots, Y_{N}$ are arbitrary real 
numbers and $Y_{n}, n=0,1, \cdots$, satisfies a homogeneous, linear difference equation of order $N+1$ with real, constant coefficients

$$
\sum_{r=0}^{N+1} a_{r} Y_{n+N+1-r}=0 \quad\left(a_{0} a_{N+1} \neq 0\right),
$$

then the generating function of $Y_{n}$ is given by

$$
\sum_{n=0}^{\infty} Y_{n} t^{n}=\sum_{j=0}^{N}\left(\sum_{r=0}^{j} a_{r} Y_{j-r}\right) t^{j} / \sum_{r=0}^{N+1} a_{r} t^{r} \quad(N=0,1, \cdots),
$$

we obtain from (2.3) with $N=m-1, a_{0}=1, a_{1}=-2 x, a_{r}=0, r=2,3$, $\cdots, m-1$, and $a_{m}=b^{(m-2) / 2}$, the generating function for $R_{n}$,

$$
\frac{1+(2(1-m) / m) x t}{1-2 x t+b^{(m-2) / 2} t^{m}}=\sum_{n=0}^{\infty} t^{n} R_{n}(x, m, b) .
$$

For $m=2,(2.4)$ reduces to the generating function for $T_{n}(x)$.

It is now possible to express $R_{n}$ in terms of $P_{n}$. In (1.1), set $p=-1$, $C=1$, and replace $x$ by $2 x / m$ to obtain

$$
1 /\left(1-2 x t+y t^{m}\right)=\sum_{n=0}^{\infty} t^{n} P_{n}(m, 2 x / m, y,-1,1) .
$$

Set $y=b^{(m-2) / 2}$ in (2.5) and then multiply both sides by $1+(2(1-m) / m) x t$ to obtain, noting (2.4),

$$
\begin{aligned}
R_{n}(x, m, b)= & P_{n}\left(m, 2 x / m, b^{(m-2) / 2},-1,1\right) \\
& +(2(1-m) / m) x P_{n-1}\left(m, 2 x / m, b^{(m-2) / 2},-1,1\right) \\
& (n=1,2, \cdots) .
\end{aligned}
$$

For $m=2,(2.6)$ gives

$$
\begin{array}{r}
T_{n}(x)=P_{n}(2, x, 1,-1,1)-x P_{n-1}(2, x, 1,-1,1) \\
\quad(n=1,2, \cdots) .
\end{array}
$$

Conversely, we have

$$
P_{n}\left(m, 2 x / m, b^{(m-2) / 2},-1,1\right)=\sum_{j=0}^{n}(2 x(m-1) / m)^{n-j} R_{j}(x, m, b) ;
$$

and for $m=2,(2.8)$ gives

$$
C_{n}^{(1)}(x)=U_{n}(x)=\sum_{j=0}^{n} x^{n-j} T_{j}(x) .
$$


In $[1$, p. $702,(3.5)]$, it was shown that

$$
D_{x}^{k} P_{n+k}(m, x, y, p, C)=k !(-m)^{k}\left(\begin{array}{l}
p \\
k
\end{array}\right) P_{n}(m, x, y, p-k, C)
$$

$$
\left(D_{x}^{k}=d^{k} / d x^{k}\right)
$$

Applying (1.2) to (2.10), we obtain, after simplification,

$$
\begin{aligned}
& m D_{x}^{k} R_{n+k}(x, m, C / y)=(k-1) !(n+k) 2^{k}(C / y)^{n / 2} \\
& \cdot C^{k} P_{n}\left(m, 2 x(C y)^{1 / 2} / m, y,-k, C\right) \quad(k=1,2, \cdots) .
\end{aligned}
$$

Since $P_{n}(2, x, 1,-k, 1)=C_{n}^{(k)}(x)$, the Gegenbauer polynomial, we obtain from (2.11)

$$
D_{x}^{k} T_{n+k}(x)=(k-1) !(n+k) 2^{k-1} \cdot C_{n}^{(k)}(x) \quad(k=1,2, \cdots)
$$

Using (1.3), we note that

$$
C^{k} P_{n}\left(m, 2 x(C y)^{1 / 2} / m, y,-k, C\right)=P_{n}\left(m, 2 x(y / C)^{1 / 2} / m, y / C,-k, 1\right),
$$

and thus, (2.11), with $b=C / y$, becomes

$$
m D_{x}^{k} R_{n+k}(x, m, b)
$$

$=(k-1) !(n+k) 2^{k} b^{n / 2} P_{n}\left(m, 2 x b^{-1 / 2} / m, 1 / b,-k, 1\right) \quad(k=1,2, \cdots)$; and for $n=0,(2.13)$ gives

$$
D_{x}^{k} R_{k}(x, m, b)=k ! 2^{k} / m \quad(k=1,2, \cdots) .
$$

The polynomials $R_{n}$ also satisfy the following recurrence relations, where $R_{n}{ }^{\prime}=D_{x} R_{n}$ :

$$
\begin{aligned}
R_{n}^{\prime}= & (x / n) R_{n}^{\prime} \\
& -((m / 2) /(n-m+1)) b^{(m-2) / 2} R_{n-m+1}^{\prime}, \\
2 R_{n}= & (1 /(n+1)) R_{n+1}^{\prime} \\
& -((m-1) /(n-m+1)) b^{(m-2) / 2} R_{n-m+1}^{\prime}, \\
R_{n}= & ((m / 2) /(n+1)) R_{n+1}^{\prime}-((m-1) x / n) R_{n}^{\prime}, \\
\left(2(m-1) x^{2} / n\right) R_{n}^{\prime}= & 2(m-1) x R_{n} \\
& +\left(\left(m^{2} / 2\right) /(n-m+2)\right) b^{(m-2) / 2} R_{n-m+2}^{\prime} \\
& -m b^{(m-2) / 2} R_{n-m+1} .
\end{aligned}
$$


Relations (2.15), $\cdots,(2.18)$ were obtained, respectively, by applying (1.2) to $[1$, p. $700,(2.5),(2.6),(2.7),(2.8)]$.

3. An expansion theorem. In [1, pp. 709-710], a theorem was given for the expansion of a polynomial of degree $s$ in $x$ as a linear combination of generalized Humbert polynomials. A similar theorem is now given for an expansion in terms of the derived polynomials $R_{n}$.

THEOREM 1. Let $f(x)=\sum_{n=0}^{s} A_{n} x^{n}$ be an arbitrary polynomial of degree $s$ in $x$. Then $f(x)$ may be expressed in terms of the polynomials $R_{n}$ by the formula

$$
f(x)=K_{0} / m+\sum_{n=1}^{8} K_{n} R_{n}(x, m, b),
$$

where

$$
\begin{aligned}
K_{n}=m \quad \sum_{k=0}^{[(s-n) / m]}\left(\begin{array}{c}
n+m k \\
k
\end{array}\right) 2^{2-(n+m k)} b^{(m-2) k / 2} A_{n+m k} & \\
& (n=0,1, \cdots, s) .
\end{aligned}
$$

Proof. In [1, p. 709], there is stated the identity

$$
\sum_{n=0}^{8} \sum_{k=0}^{[n / m]} B_{n, k}=\sum_{n=0}^{8} \sum_{k=0}^{[(8-n) / m]} B_{n+m k, k}
$$

If we substitute for $x^{n}$ (as given by (1.5)) in to $\sum_{n=0}^{s} A_{n} x^{n}$, we obtain our result (3.1) and (3.2) by an application of (3.3).

4. Additional results. In [5, p. 135], it was shown that if the roots of the characteristic equation for the difference equation

$$
Y_{n+m}=q Y_{n+r}+p Y_{n} \quad(p q \neq 0,1 \leqq r<m, n=0,1, \cdots),
$$

are distinct, then

$$
Y_{n m}=\sum_{k=0}^{n}\left(\begin{array}{l}
n \\
k
\end{array}\right) p^{n-k} q^{k} Y_{k r} \quad(n=0,1, \cdots) .
$$

Since the characteristic equation of $(2.1)$ is

$$
z^{m}-2 x z^{m-1}+b^{(m-2) / 2}=0,
$$

then (4.2), with $Y_{n} \equiv R_{n}$ and $r=m-1$, gives

$$
\begin{array}{r}
R_{n m}(x, m, b)=\sum_{k=0}^{n}\left(\begin{array}{l}
n \\
k
\end{array}\right)\left(-b^{(m-2) / 2}\right)^{n-k}(2 x)^{k} R_{k(m-1)}(x, m, b) \\
(n=0,1, \cdots),
\end{array}
$$


which is valid provided the roots of (4.3) are distinct. For $m=2$, (4.4) gives

$$
\begin{aligned}
(-1)^{n} T_{2 n}(x)=\sum_{k=0}^{n}\left(\begin{array}{l}
n \\
k
\end{array}\right)(-2 x)^{k} T_{k}(x) & \\
& (x \neq \pm 1, n=0,1, \cdots) .
\end{aligned}
$$

Since $U_{n+2}(x)=2 x U_{n+1}(x)-U_{n}(x)$, we also have

$$
\begin{aligned}
(-1)^{n} U_{2 n}(x)=\sum_{k=0}^{n}\left(\begin{array}{l}
n \\
k
\end{array}\right) & (-2 x)^{k} U_{k}(x) \\
& (x \neq \pm 1, n=0,1, \cdots) .
\end{aligned}
$$

In $[6$, p. 253, (5.1), (5.2)], it was shown that

$$
\begin{aligned}
V_{n} & =2 a^{n / 2} T_{n}\left(d /\left(2(a)^{1 / 2}\right)\right) \quad(n=0,1, \cdots) . \\
Z_{n+1} & =a^{n / 2} U_{n}\left(d /\left(2(a)^{1 / 2}\right)\right) \quad(n=0,1, \cdots),
\end{aligned}
$$

where $Z_{n}$ and $V_{n}$ are Lucas functions which satisfy

$$
W_{n+2}=d W_{n+1}-a W_{n}, \quad d^{2}-4 a \neq 0, \quad(n=0,1, \cdots),
$$

with $d \neq 0$ and $a \neq 0$ as arbitrary real numbers; and $Z_{n} \equiv W_{n}$ if $W_{0}=0$ and $W_{1}=1$, and $V_{n} \equiv W_{n}$ if $W_{0}=2$ and $W_{1}=d$. Applying (4.7) to (4.5) and (4.8) to (4.6), we obtain, respectively,

$$
\begin{aligned}
& (-1)^{n} V_{2 n}=\sum_{k=0}^{n}(-1)^{k}\left(\begin{array}{l}
n \\
k
\end{array}\right) a^{n-k} d^{k} V_{k} \quad(n=0,1, \cdots), \\
& (-1)^{n} Z_{2 n+1}=\sum_{k=0}^{n}(-1)^{k}\left(\begin{array}{l}
n \\
k
\end{array}\right) a^{n-k} d^{k} Z_{k+1} \quad(n=0,1, \cdots) \text {. }
\end{aligned}
$$

An application of (4.2) to (4.9) gives

$$
W_{2 n}=\sum_{k=0}^{n}\left(\begin{array}{l}
n \\
k
\end{array}\right)(-a)^{n-k} d^{k} W_{k} \quad(n=0,1, \cdots) .
$$

If we apply the inverse relation pair identity

$$
A_{n}=\sum_{k=0}^{n}(-1)^{k}\left(\begin{array}{l}
n \\
k
\end{array}\right) B_{k}, \quad B_{n}=\sum_{k=0}^{n}(-1)^{k}\left(\begin{array}{l}
n \\
k
\end{array}\right) A_{k_{2}}
$$

to (4.12) and (4.11), we obtain, respectively,

$$
\begin{aligned}
d^{n} W_{n} & =\sum_{k=0}^{n}\left(\begin{array}{l}
n \\
k
\end{array}\right) a^{n-k} W_{2 k} & (n=0,1, \cdots) . \\
d^{n} Z_{n+1} & =\sum_{k=0}^{n}\left(\begin{array}{l}
n \\
k
\end{array}\right) a^{n-k} Z_{2 k+1} & (n=0,1, \cdots) .
\end{aligned}
$$


For $p=-1,(2.2)$ simplifies to $C P_{n}-m x P_{n-1}+y P_{n-m}=0, n \geqq m \geqq 1$. Thus, if the roots of $C z^{m}-m x z^{m-1}+y=0$ are distinct, we obtain from (4.2)

$$
\begin{aligned}
& P_{n m}(m, x, y,-1, C) \\
& \quad=(-C)^{-n} \sum_{k=0}^{n}(-1)^{k}\left(\begin{array}{l}
n \\
k
\end{array}\right) y^{n-k}(m x)^{k} P_{k(m-1)}(m, x, y,-1, C) ;
\end{aligned}
$$

using (4.13), we obtain from (4.16)

$$
\begin{aligned}
(m x)^{n} P_{n(m-1)} & (m, x, y,-1, C) \\
= & \sum_{k=0}^{n}\left(\begin{array}{l}
n \\
k
\end{array}\right) y^{n-k} C^{k} P_{k m}(m, x, y,-1, C) .
\end{aligned}
$$

\section{REFERENCES}

1. H. W. Gould, Inverse series relations and other expansions involving Humbert polynomials, Duke Math. J. 32 (1965), 697-712.

2. G. Szegö, Orthogonal polynomials, Amer. Math. Soc. Colloq. Publ. Vol. 23, Amer. Math. Soc., Providence, R. I., 1939; reprint 1958.

3. J. Riordan, An introduction to combinatorial analysis, Wiley, New York, 1958.

4. D. Zeitlin, Generating functions for products of recursive sequences, Trans. Amer. Math. Soc. 116 (1965), 300-315.

5. - On solutions of homogeneous, linear, difference equations with constant coefficients, Amer. Math. Monthly 68 (1961), 134-137.

6. - Power identities for sequences defined by $W_{n+2}=d W_{n+1}-c W_{n}$, Fibonacci Quart. 3 (1965), 241-256.

University of MinNesota 